\title{
Les seuils pratiques en milieu hospitalier*
}

\author{
B. AUBERT**
}

(Manuscrit reçu le $1^{\text {er }}$ juin 1994)

RÉSUMÉ Les mesures pratiques de radioprotection mises en auvre en milieu hospitalier dépendent étroitement des caractéristiques des sources de rayonnement ionisant et de leurs méthodes d'utilisation que ce soit dans un but thérapeutique ou diagnostique. Les sources sont très diverses, émettant des photons $X$ et gamma, des électrons de haute énergie ou des particules bêta. On les rencontre sous forme scellée ou de générateurs électriques, dans les services de radiothérapie, de curiethérapie et de radiodiagnostic. Dans les services de médecine nucléaire et dans les laboratoires, on utilise principalement des sources sous forme non scellée. Ainsi, le personnel selon son activité sera soumis au risque d'exposition externe ou interne. Les seuils pratiques sont souvent basés sur les valeurs de débit d'équivalent de dose adaptés aux locaux considérés : $25,7,5$ ou 2,5 $\mu \mathrm{Sv} \cdot \mathrm{h}^{-1}$. Des hypothèses surestimant les conditions d'utilisation des sources ou les temps de présence des personnes permettent en pratique d'avoir des niveaux d'exposition du personnel bien inférieurs aux limites annuelles. Ces niveaux sont évalués par la dosimétrie individuelle telle qu'elle est définie par la réglementation française. Pour les postes où le risque d'exposition est le plus élevé, une surveillance opérationnelle permet d'apprécier les parties de l'organisme les plus exposées et d'adopter des mesures de protection adéquates. Un bilan de la dosimétrie individuelle en milieu hospitalier basé à la fois sur les résultats de la surveillance réglementaire et sur les mesures obtenues par la surveillance opérationnelle est présenté.

ABSTRACT Practical aspects of radiation protection in hospitals are closely linked to the source characteristics and methods of use for diagnosis and therapy applications. Sources vary depending upon whether they emit $X$ and $\gamma$ photons, high energy electrons or beta particles. In external radiation therapy, brachytherapy and radiodiagnostic departments, sources are either sealed or electrically generated. In nuclear medicine departments and in laboratories, sources are unsealed most of the time. The personnel is thus exposed, depending on their activity, to the risk of external and/or internal exposure. Practical thresholds are often based on dose equivalent rate values suited to the type of rooms : $25,7.5$ or $2.5 \mu \mathrm{Sv} \cdot \mathrm{h}^{-1}$. Assumptions, overestimating conditions under which sources are used or personnel attendance, allow in practice to obtain exposure levels much lower than annual limits. These levels are measured by individual dosimetry as defined by french legislation. For the most exposed working situations, an additional survey allows to appreciate the most exposed part of the body and to carry appropriate protection arrangements. $A$ balance sheet of individual dosimetry in hospital based on results from legal survey and from measurements obtained by additional survey is presented.

\footnotetext{
* Communication présentée aux journées SFRP "Seuils pratiques en radioprotection", INSTN, Saclay, 7-8 décembre 1993.

** $\quad$ Service de physique, Institut Gustave-Roussy, 94805 Villejuif Cedex.
} 


\section{B. AUBERT}

\section{Introduction}

Les recommandations de la Commission internationale de protection radiologique (CIPR) [3] conduisent à adopter des limites annuelles d'exposition (LAE) mais également à améliorer la protection du personnel par l'application du concept d'optimisation, généralement appelé ALARA selon l'abréviation de l'appellation anglaise, "as low as reasonably achievable". Ainsi, en complément des LAE, on a été amené à définir diverses notions telles que : seuil d'intervention, contrainte de dose, niveau de référence, ... Dans ce document, nous allons tout d'abord examiner les considérations pratiques sur lesquelles reposent la conception, l'aménagement et l'équipement des locaux vis-à-vis de la radioprotection du personnel et cela dans les différents domaines d'application des rayonnements ionisants en milieu hospitalier. Nous présenterons ensuite les niveaux d'exposition professionnelle tels qu'on peut les connaître au travers de la surveillance réglementaire ainsi que de la surveillance opérationnelle. Cette dernière est, cependant, limitée aux postes où l'exposition de l'organisme et/ou de certains organes est la plus élevée.

\section{Considérations pratiques vis-à-vis des locaux et du matériel}

Quatre domaines sont concernés par l'utilisation des rayonnements ionisants : radiothérapie externe, curiethérapie, radiodiagnostic et médecine nucléaire, ce dernier domaine couvrant également les laboratoires. Ainsi, vis-à-vis de la radioprotection on se trouve face à :

- différents types de source : générateurs électriques (photons et électrons), sources scellées et sources non scellées,

- différents risques d'exposition : externe et interne,

- différentes conditions d'utilisation : zone contrôlée interdite au personnel durant l'irradiation du patient, zone contrôlée, zone surveillée accessible aux patients et au public.

Il en résulte qu'en pratique la radioprotection va reposer sur la conception des locaux, la conception du matériel, l'équipement des locaux et, enfin, le respect par le personnel des consignes. Nous allons parcourir les trois premiers points dans les quatre domaines cités précédemment et considérer les aspects pratiques de radioprotection qui, pour certains, peuvent être assimilés à des seuils.

\subsection{Radiothérapie externe}

Les deux appareils constituant l'équipement de base sont l'appareil de cobalthérapie et l'accélérateur linéaire (photons et/ou électrons). Ces deux appareils sont installés dans les locaux dont la structure générale est donnée dans la figure 1. La salle est "zone contrôlée interdite au personnel pendant l'irradiation" et un dispositif de sécurité arrête immédiatement l'émission de rayonnement en cas d'ouverture de la porte avant la fin de l'irradiation. 


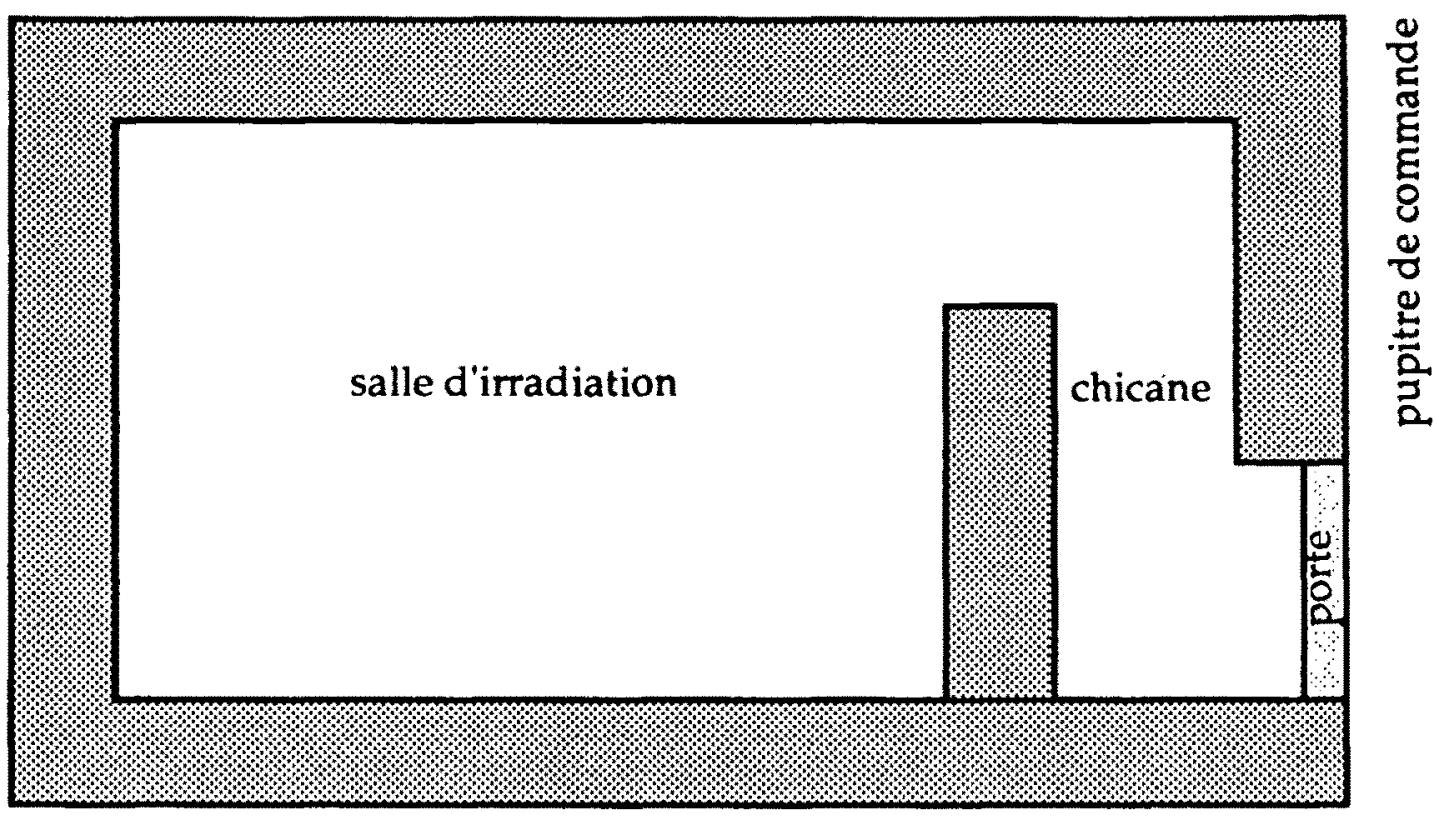

Fig. 1 - Structure d'une salle de radiothérapie externe.

Structure of a treatment room used for external radiotherapy.

L'épaisseur des parois est calculée d'après les caractéristiques d'émission et d'utilisation de l'appareil ainsi que d'après l'usage des locaux adjacents. Le tableau I précise les débits d'équivalents de dose à respecter en fonction du type de local. Les hypothèses retenues sur l'utilisation des appareils sont telles qu'elles garantissent, en pratique, un niveau d'exposition très faible du personnel et du public. En effet, outre la distance, on doit considérer la charge de travail de la machine (en gray à $1 \mathrm{~m}$ par semaine), la pondération dans les différentes orientations du faisceau et le facteur d'occupation du local pour lequel on calcule la protection.

\section{TABLEAU I}

Limites annuelles d'exposition et débits horaires considérés pour le calcul des protections en fonction de l'affectation des locaux [2]

Annual limits of exposure and rates per hour considered for protection calculation as a function of the room assignment

\begin{tabular}{|lcc|}
\hline Lieu & $\begin{array}{c}\text { Limite annuelle } \\
\text { d'exposition }(\mathbf{m S v})\end{array}$ & $\begin{array}{c}\text { Débit horaire } \\
\text { maximal } \\
(\mu \mathbf{S v} / \mathbf{h})\end{array}$ \\
\hline Zone contrôlée & 50 & 25 \\
Zone surveillée & 15 & 7,5 \\
\hline Zone non surveillée & 5 & 2,5 \\
$\cdots$ & & 25 \\
\hline
\end{tabular}


En pratique, on considère : une irradiation en continu (40 h par semaine), une pondération égale à 1 pour toutes les orientations et un facteur d'occupation égal à 1 pour les travailleurs de catégorie A. Ces trois paramètres, très largement surestimés, conduisent à des protections dont les ordres de grandeurs sont précisés dans le tableau II.

En ce qui concerne les appareils de télégammathérapie, leur conception doit assurer la protection du personnel quand la source est en position de stockage. Retenons que les normes [1] imposent que le blindage de la tête doit atténuer le rayonnement de manière telle que, dans les conditions arrêt-faisceau, le débit de dose du rayonnement parasite ne dépasse pas $0,02 \mathrm{~m} . \mathrm{Gy} \cdot \mathrm{h}^{-1}$ à $1 \mathrm{~m}$ de la source et $0,2 \mathrm{~m}$. Gy $\cdot \mathrm{h}^{-1}$ en tout point facilement accessible situé à $5 \mathrm{~cm}$ du blindage.

\section{TABLEAU II}

Ordre de grandeur des épaisseurs des parois des salles d'irradiation en radiothérapie externe, pour du béton ordinaire (densité $: 2,35 \mathrm{~g} . \mathrm{cm}^{-3}$ ) Rough estimates of wall thicknesses in treatment rooms for external therapy $\left(-\right.$ concrete density $=2.35 \mathrm{~g} \cdot \mathrm{cm}^{-3}$ )

\begin{tabular}{|lcc|}
\hline Appareil & Faisceau primaire & $\begin{array}{c}\text { Rayonnement } \\
\text { diffusé et de fuite }\end{array}$ \\
\hline Cobalthérapie & $1,2 \mathrm{~m}$ & $0,5 \mathrm{~m}$ \\
\hline Accélérateur linéaire & $2,5 \mathrm{~m}$ & $1,5 \mathrm{~m}$ \\
\hline
\end{tabular}

\subsection{Curiethérapie}

Dans cette application, les sources sont placées dans le patient durant quelques jours pour les applications dites à bas débit de dose. Deux types de source sont principalement utilisés, le césium 137 et l'iridium 192. Ces deux radionucléides ont apporté une amélioration vis-à-vis de l'exposition externe du personnel, par rapport au radium 226 précédemment utilisé. Le tableau III permet de comparer le débit d'équivalent de dose à 1 mètre pour ces trois radionucléides.

Pour le césium 137, l'utilisation de projecteur de sources, grâce auquel on peut commander à distance la mise en place ou le stockage des sources, garantit une protection quasi parfaite. Par contre, la préparation et la mise en place des fils d'iridium, puis les soins au patient pendant la période d'hospitalisation sont des phases qui exposent inévitablement le personnel, malgré les dispositifs de protection adaptés (pinces, écrans plombés, stockeurs, ...).

En ce qui concerne l'hospitalisation, la conception des chambres, telle que celle qui est présentée dans la figure 2 [4], permet, grâce à un refend en maçonnerie, de limiter le débit d'exposition dans le couloir à $7 \mu \mathrm{Sv} . \mathrm{h}^{-1}$ pour une source de césium 137 présentant un débit de dose dans l'air de $500 \mu \mathrm{Gy} \cdot \mathrm{h}^{-1} \cdot \mathrm{m}^{-2}$. 
TABLEAU III

Comparaison des débits d'équivalent de dose dans le cas d'une source ponctuelle de 1 GBq (27 mCi) d'iridium 192, de césium 137 et de radium 226 située à $1 \mathrm{~m}$ Comparison of dose equivalent rates for a point source of 1 GBq $(27 \mathrm{mCi})$ of iridium 192, caesium 137 and radium 226 at a distance of $1 \mathrm{~m}$

\begin{tabular}{|l|c|}
\hline Radionucléide & Debit dequivalent de dose \\
iridium 192 & 121 \\
césium 137 & 86,2 \\
radium 226 & 215 \\
\hline
\end{tabular}

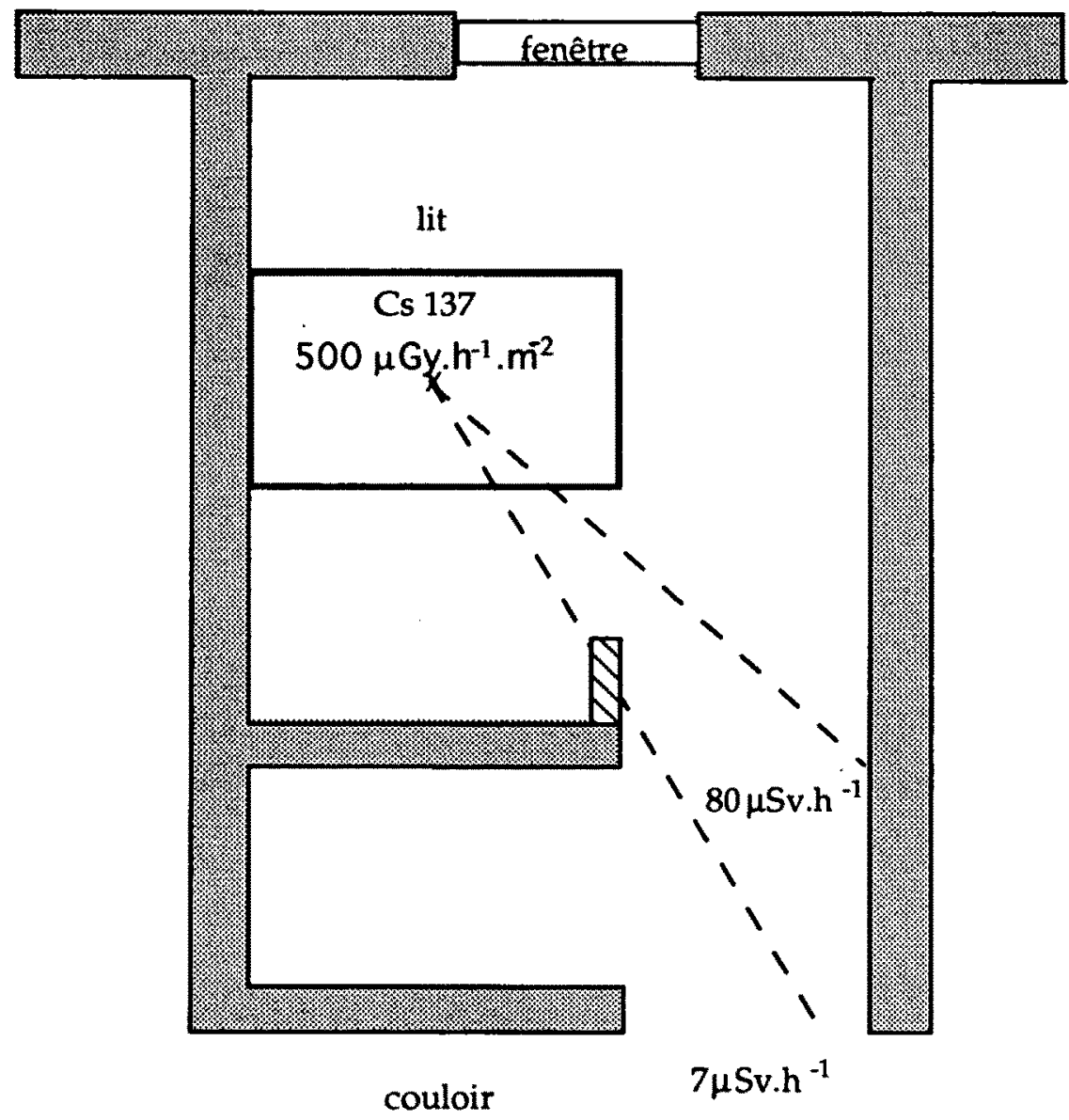

Fig. 2 - Exemple de chambre accueillant des malades porteurs de sources radioactives, d'après [4].

Example of a treatment room for patients undergoing brachytherapy.

Récemment, est apparue la technique de curiethérapie à haut débit qui met en ouvre une source d'iridium 192 de $370 \mathrm{GBq}(10 \mathrm{Ci})$. Les temps d'application de la source sont de quelques secondes et l'implantation de l'appareil est similaire à celle d'un appareil de télégammathérapie. 


\subsection{Radiodiagnostic}

Les générateurs, les tubes et les gaines des appareils de radiodiagnostic font l'objet d'une homologation [7-8] qui garantit, en particulier, le niveau de fuite des gaines. Cette disposition concerne surtout la protection des patients mais n'est pas sans influence sur celle du personnel. Pour les installations conventionnelles, des murs en briques pleines de $20 \mathrm{~cm}$ ou des écrans de $2 \mathrm{~mm}$ de plomb sur $2 \mathrm{~m}$ de hauteur garantissent la protection des locaux adjacents. Dans la salle elle-même, le personnel se trouve derrière des paravents en plomb et verre plombé.

Certaines pratiques nécessitent la présence de l'opérateur près du patient. Tout un ensemble d'accessoires (tablier, chasuble, cache-thyroïde, lunettes, gants, ...), présentant une épaisseur d'équivalent de plomb d'au moins $0,35 \mathrm{~mm}$, assurent une protection efficace vis-à-vis du rayonnement diffusé. Néanmoins, dans certaines situations, par exemple en radiologie interventionnelle, l'opérateur ne peut se munir de gants plombés et le risque d'exposer les doigts au rayonnement primaire n'est pas nul. Il est important de noter que le débit de ce rayonnement est environ 1000 fois supérieur à celui du rayonnement diffusé.

\subsection{Médecine nucléaire}

Cette application des rayonnements ionisants se caractérise, comme pour les laboratoires, par l'utilisation de sources non scellées, d'où un risque additionnel d'exposition interne.

La radioprotection repose alors sur une hiérarchisation des locaux en fonction du niveau d'activité, et par un aménagement et un équipement destiné à éviter, ou au moins à limiter, les conséquences d'une contamination radioactive. Les manipulations de produits volatils, tels que les isotopes de l'iode, se font dans des enceintes closes, parfois blindées, reliées à l'extérieur par une cheminée munie d'un filtre. Les effluents aqueux sont traités comme rejets contrôlés. L'arrêté du 30 octobre 1981 spécifie dans l'article 8 que l'évacuation des effluents liquides ne peut intervenir que si l'activité volumique est inférieure à $7 \mathrm{~Bq} \cdot \mathrm{l}^{-1}$. En pratique cette disposition ne s'applique qu'aux effluents de nature inconnue. Pour les effluents hospitaliers parfaitement identifiés, il convient d'appliquer les dispositions figurant dans l'avis aux utilisateurs de radioéléments [5]. Celui-ci précise d'une part que la quantité totale rejetée par an doit être inférieure à $37 \mathrm{GBq}$ et d'autre part que la quantité journalière rejetée diluée dans le volume total moyen d'eaux usées rejetées en $24 \mathrm{~h}$ par l'établissement ne doit pas dépasser une certaine concentration. Actuellement, compte tenu de l'évolution des grandeurs utilisées en radioprotection (CMA remplacées par LAI), les valeurs de concentration à utiliser sont égales aux valeurs des limites annuelles d'incorporation (LAI) par ingestion exprimées en $\mathrm{Bq} \cdot \mathrm{m}^{-3}$.

Les manipulations de sources en flacon ou en seringue s'effectuent en se protégeant les doigts par des protège-flacons et des protège-seringues, ces der- 
niers offrant pour le technétium $99 \mathrm{~m}$ un facteur d'atténuation de l'ordre de 100. Il est à noter que l'utilisation de verre au plomb et de tungstène a facilité et favorisé l'usage de ces dispositifs.

\section{Résultats de la surveillance individuelle}

\subsection{Surveillance réglementaire}

\subsubsection{Exposition externe}

En France, la réglementation prévoit une surveillance effectuée selon les modalités suivantes : film dosimètre "poitrine" pour la surveillance de l'organisme de tout travailleur de catégorie A et film dosimètre "poignet" pour tout travailleur de catégorie A soumis à un risque d'exposition externe des mains. Ces films dosimètres sont portés pendant un mois.

Les tableaux suivants fournissent quelques données sur l'exposition des travailleurs du milieu médical telle qu'elle apparaît au travers des résultats des films dosimètres. Le tableau IV présente la répartition des équivalents de dose annuels relatifs à l'exposition externe pour le personnel du milieu médical en France en 1992, d'après les données du Service central de protection contre les rayonnements ionisants (SCPRI), ainsi que celle concernant le personnel de l'institut Gustave-Roussy (IGR) pour 1992 et sur 11 ans de 1982 à 1992.

Ces données montrent clairement que, globalement, plus de $99 \%$ des travailleurs de catégorie A sont soumis à des expositions annuelles dont le niveau est inférieur à $5 \mathrm{mSv}$, valeur d'équivalent de dose annuel pour le public.

TABLEAU IV

Répartition des équivalents de dose annuels (exposition externe) pour le personnel du milieu médical en France et à l'IGR (source SCPRI) Distribution of annual dose equivalents (external exposure) for the medical personnel in France and at IGR

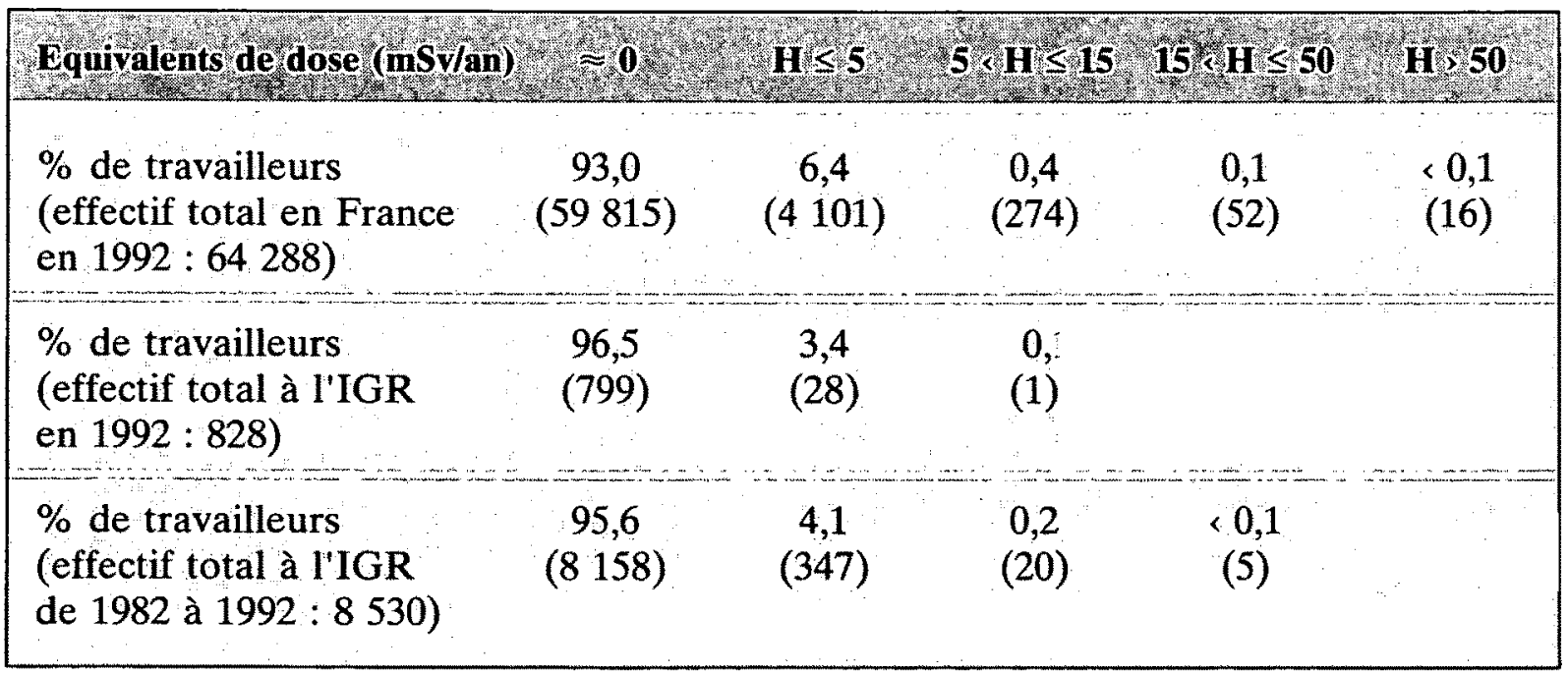


Afin de mieux analyser les postes où les risques d'exposition sont les plus élevés, nous avons porté dans le tableau $\mathrm{V}$ les valeurs moyennes de l'équivalent de dose annuel des travailleurs les plus exposés. Il est à noter que les résultats concernant la radiologie ont été classés en deux catégories, avant et à partir de 1990, date de l'installation de la radiologie interventionnelle. Entre ces deux périodes, la valeur moyenne de l'équivalent de dose a augmenté d'un facteur 16, ce qui situe cette activité comme une des pratiques hospitalières conduisant aux risques d'exposition externe les plus élevés. Cette constatation va se trouver confirmée par les mesures de l'exposition aux doigts dans le cadre de la surveillance opérationnelle.

TABLEAU V

Valeur moyenne et gamme de l'équivalent de dose annuel des travailleurs occupant les postes les plus exposés à l'Institut Gustave-Roussy, de 1982 à 1992 Annual dose equivalent mean values and ranges for the most exposed workers at IGR, 1982-1992

\begin{tabular}{|c|c|c|c|c|c|}
\hline \multicolumn{6}{|c|}{$\begin{array}{ccc}\text { Service } & \text { Physique } & \text { Médecine } \\
\text { nucleaire }\end{array}$ Curiethérapie } \\
\hline activité & $\begin{array}{l}\text { préparation } \\
\text { de sources } \\
\text { non scellées }\end{array}$ & $\begin{array}{l}\text { injection } \\
\text { de produits } \\
\text { radioactifs }\end{array}$ & $\begin{array}{l}\text { préparation } \\
\text { et utilisation } \\
\text { de sources } \\
\text { d'iridium }\end{array}$ & $\begin{array}{l}\text { conven- } \\
\text { tionnel }\end{array}$ & $\begin{array}{l}\text { conven- } \\
\text { tionnel et } \\
\text { interven- } \\
\text { tionnel }\end{array}$ \\
\hline $\begin{array}{l}\text { equivalent } \\
\text { de dose } \\
\text { par travailleurs } \\
\text { (mSv/an) }\end{array}$ & 1,6 & 3,3 & 1,7 & 0,4 & 6,4 \\
\hline $\begin{array}{l}\text { gamme } \\
\text { (mSv/an) }\end{array}$ & $0,2-4,9$ & $0,35-16,7$ & $0,2-5,65$ & $0,2-1,4$ & $0,2-38,4$ \\
\hline
\end{tabular}

Néanmoins, toutes les pratiques hospitalières n'ont pas évolué dans le sens de l'accroissement de l'exposition. La curiethérapie nous en fourni un exemple au travers des résultats du tableau VI montrant l'amélioration de l'exposition externe depuis 1954. Cette amélioration spectaculaire est due à la mise en œuvre de nouvelles techniques (préparation non radioactive, projecteur de sources) et de nouvelles sources (iridium 192 et césium 137) à la place du radium 226 .

\subsubsection{Exposition interne}

La réglementation française prévoit tous les 6 mois pour tout travailleur de catégorie $\mathrm{A}$, une analyse radiotoxicologique portant sur les urines recueillies durant $24 \mathrm{~h}$ et un examen anthropogammamétrique. Ce dernier examen ne s'adresse qu'à certains travailleurs de catégorie A (ceux manipulant des activi- 


\section{TABLEAU VI}

Amélioration de l'équivalent de dose annuel (mSv/an) reçu par le personnel de curiethérapie, due à l'introduction de nouvelles techniques et de nouvelles sources

Improvement of the annual dose equivalent $(\mathrm{mSv} / \mathrm{y})$ received by brachytherapy personnel, with the use of new techniques and new sources

\begin{tabular}{|cccccc|}
\hline Activité & $\begin{array}{c}1954^{*} \\
\text { radium }\end{array}$ & $\begin{array}{c}1962 \\
\text { appl. non } \\
\text { radioactive }\end{array}$ & $\begin{array}{c}1971 \\
\text { projecteur } \\
\text { de sources }\end{array}$ & $\begin{array}{c}1976 \\
\text { arrêt } \\
\text { du radium }\end{array}$ & $\begin{array}{c}1981 \\
\text { et après }\end{array}$ \\
\hline médecin & 88 & 14,4 & 4,3 & 1,8 & $\approx 0$ \\
\hline manipulateur & $309 * *$ & 48,1 & 8,1 & 5,2 & 3,1 \\
\hline infirmière & & 2,4 & 1,4 & 0,5 & $\approx 0$ \\
\hline hôtellerie & 2,8 & 2,1 & $\approx 0$ & $\approx 0$ \\
\hline
\end{tabular}

* LAE $=150 \mathrm{mSv} / \mathrm{an}$

** manipulateurs à ce poste seulement 6 mois/an

tés relativement élevées, $\geq 0,1 \mathrm{GBq}$, de radionucléides émetteurs $\gamma$ ) et il peut être complété par un comptage thyrö̈dien pour le personnel manipulant des isotopes radioactifs de l'iode. Les résultats que nous présentons concernent les personnes les plus exposées aux risques d'exposition interne, c'est-à-dire les techniciens qui préparent les sources radioactives et administrent des solutions d'iode 131. La figure 3 présente le bilan des examens urinaires réalisés sur ces techniciens.

Compte tenu de la quantité élevée d'iode 131 manipulée dans notre établissement, ces techniciens bénéficient également d'une surveillance réglementaire complémentaire : un comptage corps entier et un comptage thyroïdien tous les 6 mois. La figure 4 présente le bilan des comptages corps entier sur les 3 dernières années.

\subsection{Surveillance opérationnelle}

\subsubsection{Exposition externe}

Les parties du corps qui sont plus particulièrement exposées sont :

- les mains pour les manipulateurs et les infirmières en médecine nucléaire, et pour les manipulateurs en curiethérapie,

- les mains, les yeux et la thyroïde pour les médecins en radiologie interventionnelle.

Les dosimètres thermoluminescents (TL) (disque ou bandelette) sont tout à fait adaptés pour l'évaluation des équivalents de dose associés à ces expositions. 


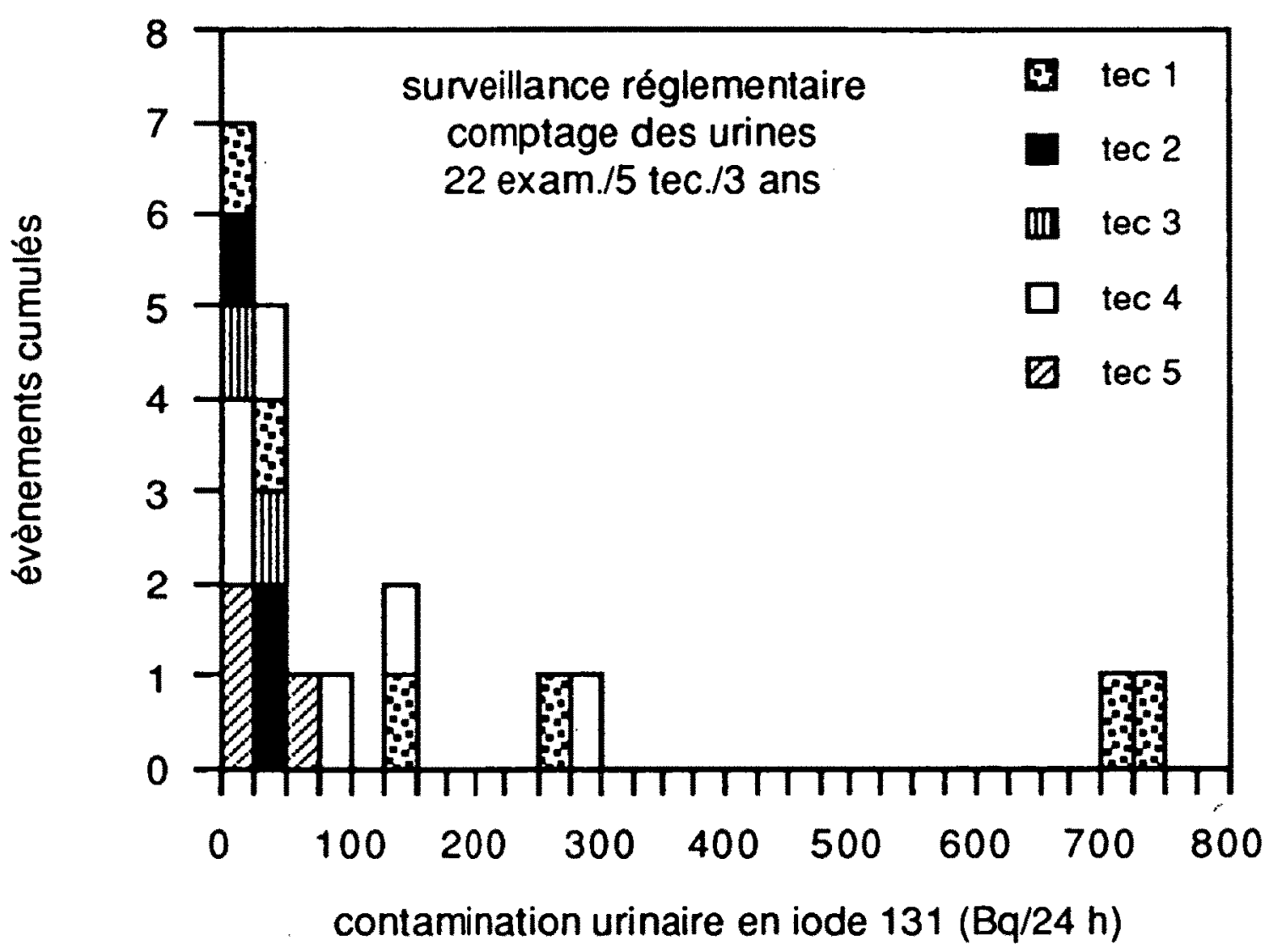

Fig. 3 - Histogramme de la contamination urinaire déterminée par la surveillance radiotoxicologique réglementaire des techniciens chargés des administrations des solutions d'iode 131 . En fait, les résultats relatifs au technicien 1 concernent une contamination qui a été suivie au cours du temps par plusieurs examens.

Urinary contamination assessed by the regulatory radiotoxicological monitoring of the technicians in charge of iodine 131 solution administration. For technician 1 , the results are related to a contamination monitored by examinations over several months.

Le tableau VII montre les résultats aux doigts les plus exposés pour les techniciens préparant chaque jour $20 \mathrm{GBq}$ (à peu près $500 \mathrm{mCi}$ ) de diverses solutions de molécules marquées au technétium $99 \mathrm{~m}$, pour les infirmières injectant ces molécules et pour les techniciens préparant et manipulant les sources d'iridium. La dernière colonne indique l'équivalent de dose moyen par an et par technicien, en considérant le fait que, pour chacun des postes, plusieurs personnes (entre 3 et 5 ) se répartissent le travail.

Le tableau VIII donne les mesures d'exposition effectuées lors de procédures éloignées et de procédures rapprochées (drainage urinaire ou biliaire par exemple) en radiologie interventionnelle. Ces mesures ont été réalisées à l'aide de dosimètres thermoluminescents placés sur les doigts, le cou (thyroïde), le front (cristallin) des radiologues. Ces résultats soulignent la nécessité d'une surveillance opérationnelle spécifique pour cette application médicale. 


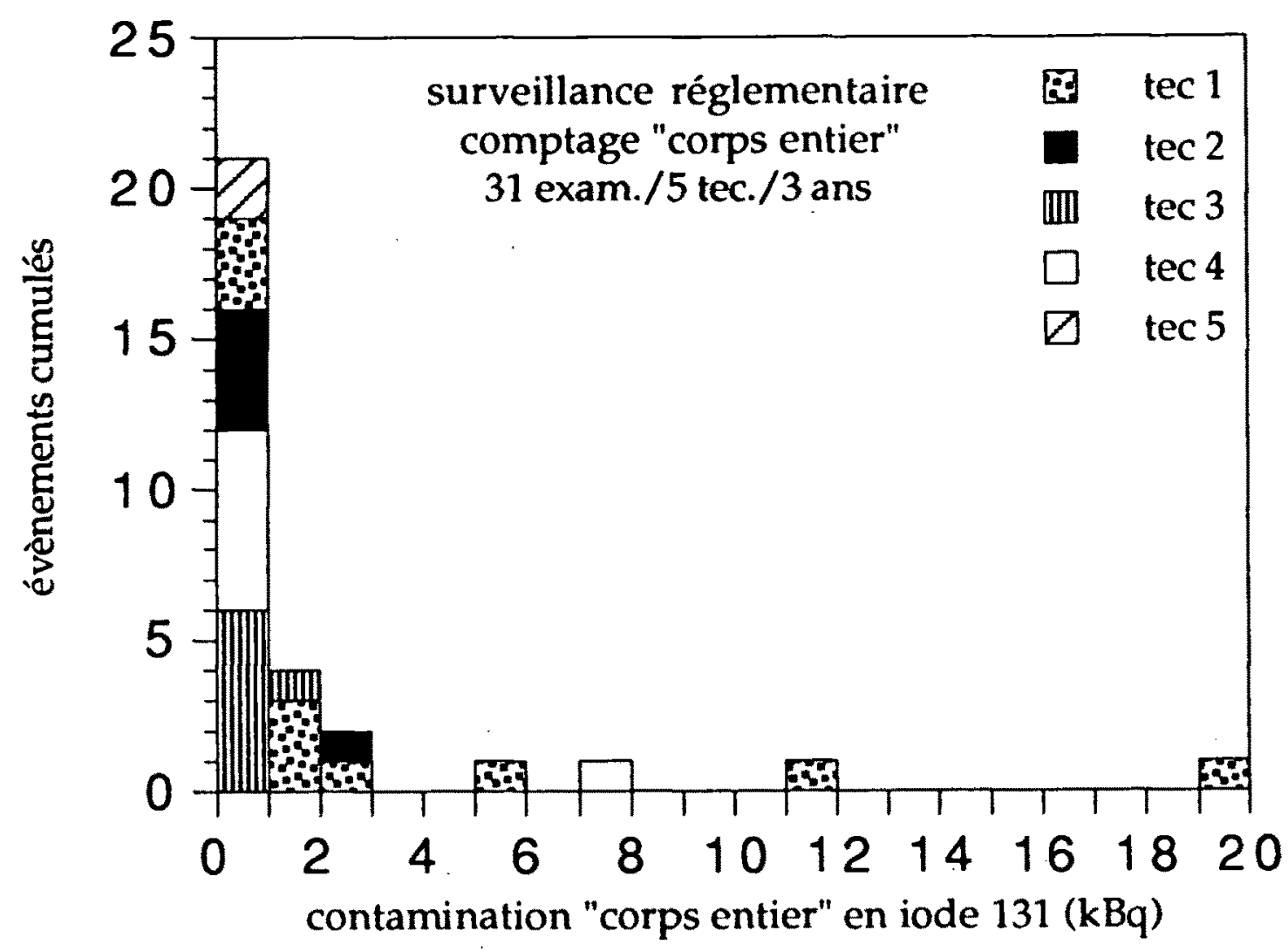

Fig. 4 - Histogramme de la contamination "corps entier" (kBq) des techniciens chargés des administrations d'iode 131. Comme dans la figure 3, la contamination du technicien 1 a été suivie au cours du temps par plusieurs examens.

Whole-body counting $(\mathrm{kBq})$ of technicians in charge of iodine 131 administration. Technician 1 was monitored over several months.

\section{TABLEAU VII}

Niveaux d'exposition des mains des travailleurs les plus exposés dans les services de physique, de médecine nucléaire et de curiethérapie Exposure levels of the hands of the most exposed workers in physics, nuclear medicine and brachytherapy departments

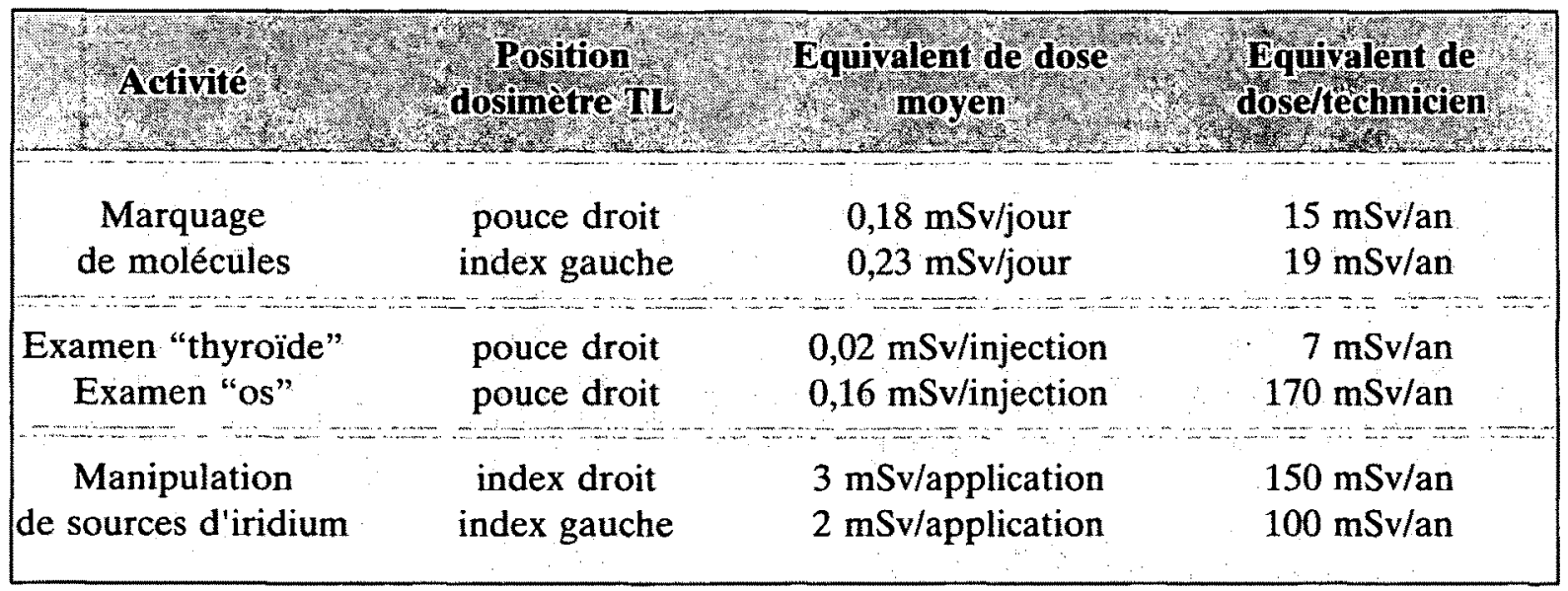


TABLEAU VIII

Niveaux d'exposition des mains, du cou et du front en radiologie interventionnelle pour des procédures éloignées et rapprochées

Mean levels of exposure of hands, neck and forehead in interventional radiology for remote and close procedures

\begin{tabular}{|lcccc|}
\hline $\begin{array}{c}\text { Position } \\
\text { des dosimetres }\end{array}$ & Main gauche & Main droite & $\begin{array}{c}\text { Cou } \\
\text { (thyroide) }\end{array}$ & $\begin{array}{c}\text { Front } \\
\text { (cristallin) }\end{array}$ \\
\hline Procédures éloignées & $1,2 \mathrm{mSv}$ & $1,4 \mathrm{mSv}$ & $0,3 \mathrm{mSv}$ & $0,2 \mathrm{mSv}$ \\
\hline Procédures rapprochées & $37,1 \mathrm{mSv}$ & $24,1 \mathrm{mSv}$ & $7,4 \mathrm{mSv}$ & $3,3 \mathrm{mSv}$ \\
\hline
\end{tabular}

\subsubsection{Exposition interne}

La surveillance opérationnelle de l'exposition interne est pratiquée dans notre institut sur une base mensuelle, afin de déterminer la fixation en iode de la thyroïde des techniciens administrant les activités thérapeutiques. Les résultats sont rapportés dans la figure 5 . Si l'on considère la demi-vie de l'iode 131 , cette fréquence mensuelle est mieux adaptée à la surveillance des travailleurs que la fréquence de 6 mois prévue dans le cadre de la surveillance réglementaire.

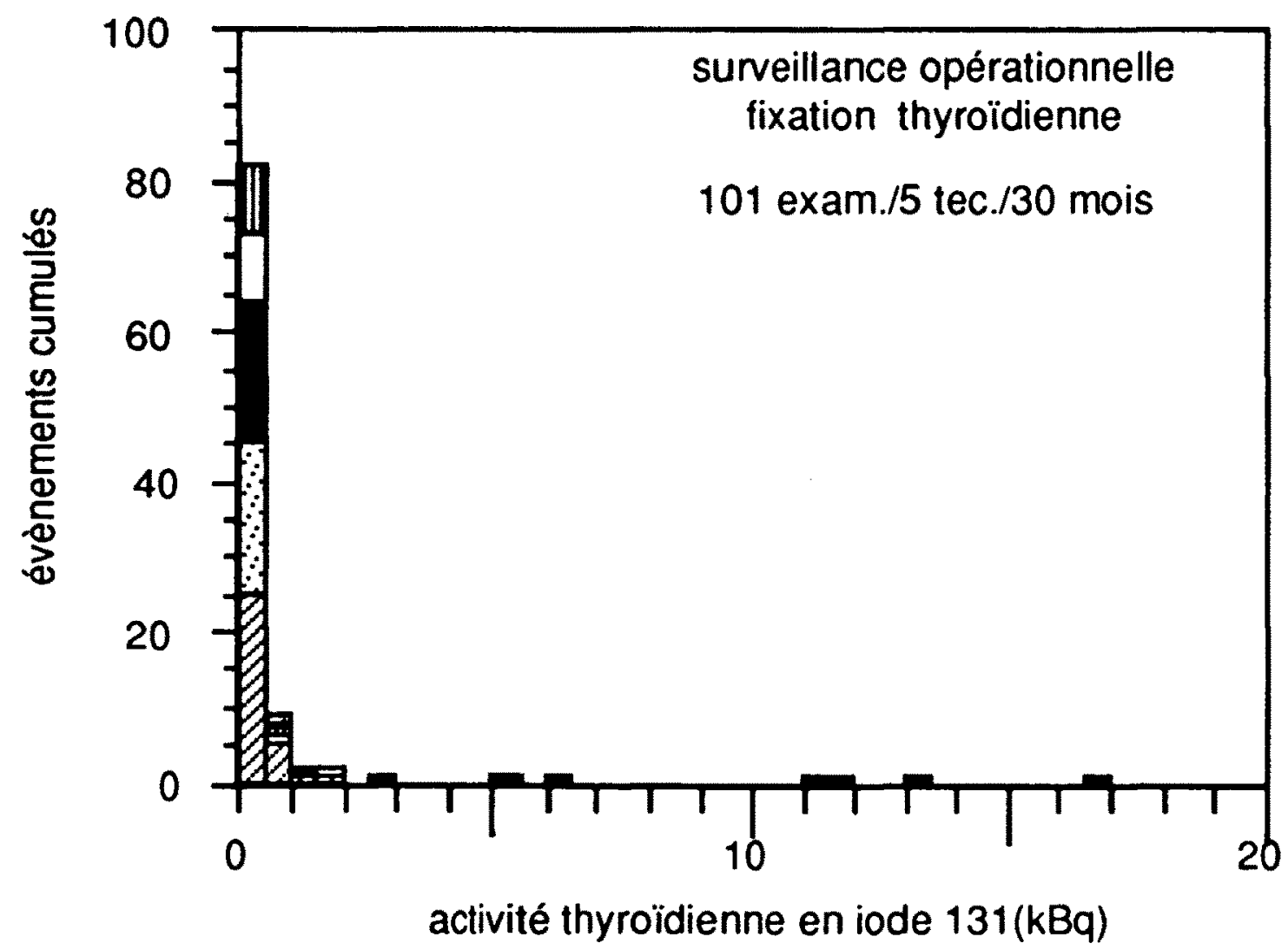

Fig. 5 - Histogramme de la fixation thyroïdienne $(\mathrm{kBq})$ en iode 131 des techniciens chargés des administrations d'iode. Ces fixations ont été mesurées avec le dispositif pour patient.

Thyroid uptakes of iodine $131(\mathrm{kBq})$ by technicians in charge of iodine administration (measured with the device for patients). 


\section{Conclusion}

Les seuils pratiques, tels qu'ils sont appliqués en milieu hospitalier, pour l'exposition externe, conduisent à une situation tout à fait satisfaisante : plus de $95 \%$ des expositions individuelles sont inférieures à la limite du public, soit $5 \mathrm{mSv}$ par an.

Les résultats obtenus par la surveillance opérationnelle montrent qu'une attention toute particulière doit être portée à quelques postes de travail et plus particulièrement à ceux où l'opérateur est proche de la "source". Si les dosimètres thermoluminescents sont parfaitement adaptés à la mesure de la dose en des points particuliers de l'organisme, leur lecture différée en limite quelque peu l'intérêt. L'évolution des dosimètres électroniques, et en particulier l'utilisation de détecteurs de petite taille, doit par contre permettre d'accéder immédiatement à des informations (seuils, débits, dose) très utiles pour l'analyse de postes et de pratiques.

Si la notion de contrainte de dose et les nouvelles limites de la CIPR 60 ne doivent pas soulever d'importants problèmes de radioprotection en milieu hospitalier, il convient d'établir des valeurs de référence. Les valeurs suivantes peuvent être retenues pour l'exposition externe :

$-10 \mathrm{mSv} / \mathrm{an}$ pour les techniques fluoroscopiques où l'opérateur est près du patient et du faisceau de rayons $X$,

$-5 \mathrm{mSv} / \mathrm{an}$ pour la manipulation de sources scellées ou non scellées,

$-2 \mathrm{mSv} / \mathrm{an}$ pour les autres applications médicales des rayonnements ionisants.

Mais doit-on considérer ces valeurs comme des contraintes, des niveaux, des seuils pratiques ...?

\section{RÉFÉRENCES}

[1] ASSOCIATION FRANÇAISE DE NORMALISATION (AFNOR) - Les équipements de télégammathérapie (Norme NF C 74-203). Paris : AFNOR, 1988.

[2] CENTRE NATIONAL DE L'ÉQUIPEMENT HOSPITALIER (CNEH). - Conseils pour l'élaboration d'un service de radiothérapie (Cahier $n^{\circ}$ 27). Paris : $\mathrm{CNEH}, 1984$.

[3] COMMISSION INTERNATIONALE DE PROTECTION RADIOLOGIQUE (CIPR). Recommandations 1990... (Publication CIPR 60). Oxford : Pergamon Press, 1991.

[4] DUTREIX A., MARINEllo G., WANBERSIE A. - Dosimétrie en curiethérapie. Paris : Masson, 1982.

[5] FRANCE - Avis aux utilisateurs de radioéléments soumis au régime d'autorisation prévu par le code de la santé publique, relatif à l'élimination des déchets radioactifs (sources non scellées exclusivement). JORF, 6 juin 1970. In : Protection contre les rayonnements ionisants (Brochure $\mathrm{n}^{\circ}$ 1420). Paris : Journaux Officiels, 1994, 173-178.

[6] FRANCE - Arrêté du 30 octobre 1981 relatif aux conditions d'emploi des radioéléments artificiels utilisés en sources non scellées à des fins médicales. JORF, 29 novembre 1981. In : 


\section{B. AUBERT}

Protection contre les rayonnements ionisants (Brochure $n^{\circ} 1420$ ). Paris : Journaux Officiels, 1994, 273-276.

[7] FRANCE - Arrêté du 9 décembre 1982 relatif à l'homologation des produits et appareils à usage préventif, diagnostique et thérapeutique. JORF, 6 juin 1970. In : Protection contre les rayonnements ionisants (Brochure $n^{\circ} 1420$ ). Paris : Journaux Officiels, 1994, 183-187.

[8] FRANCE - Circulaire du 20 avril 1986 relative à l'homologation des produits et appareils à usage préventif, diagnostique et thérapeutique. JORF, 25 mai 1986. In : Protection contre les rayonnements ionisants (Brochure $n^{\circ} 1420$ ). Paris : Journaux Officiels, 1994, 211-216. 